\title{
Comparison of three copromicroscopic methods to assess albendazole efficacy against soil-transmitted helminth infections in school-aged children on Pemba Island
}

\author{
Marco Albonico ${ }^{a, b}$, Laura Rinaldic,*, Sonia Sciasciaa, Maria E. Morgoglionec, Monica Piemonte ${ }^{c}$, Maria P. Maurellic, \\ Vincenzo Musellad, Jürg Utzingere,f, Said M. Alig, Shaali M. Ameg and Giuseppe Cringolic
}

\begin{abstract}
${ }^{a}$ Department of Biology, University of Torino, 10122 Torino, Italy; ${ }^{b}$ Ivo de Carneri Foundation, Viale Monza 44, 20127 Milano, Italy; 'Department of Veterinary Medicine and Animal Productions, University of Naples Federico II, Via della Veterinaria 1, 80137 Naples, Italy; ${ }^{d}$ Department of Health Sciences, University Magna Graecia of Catanzaro, Viale Europa, 88100 Catanzaro, Italy; ${ }^{\circledR}$ Department of Epidemiology and Public Health, Swiss Tropical and Public Health Institute, PO Box, CH-4002, Basel, Switzerland; ${ }^{\prime}$ University of Basel, PO Box, CH-4003 Basel, Switzerland; '9Public Health Laboratory-Ivo de Carneri, Pemba, Tanzania
\end{abstract}

*Corresponding author: Tel: +39 81 2536281; Fax: +39 81 2536282; E-mail: Irinaldi@unina.it

Received 6 March 2013; revised 24 May 2013; accepted 28 May 2013

\begin{abstract}
Background: The diagnostic accuracy of three faecal egg count techniques (Kato-Katz, McMaster and FLOTAC) to assess albendazole efficacy against soil-transmitted helminth (STH) infections was compared.

Methods: The study is registered with Current Controlled Trials [identifier: ISRCTN90088840]. During September November 2009, 304 school-aged children on Pemba Island, Tanzania, were screened and those infected with Ascaris lumbricoides, hookworm or Trichuris trichiura were treated with a single dose of albendazole (400 mg). Twenty-one days post-treatment, children provided a single stool sample which was examined using the same diagnostic methods. All stool samples were divided into two aliquots and one was fixed in 5\% formalin and examined using FLOTAC and McMaster approximately 6 months after collection.

Results: Using fresh stool samples, comparable prevalences were demonstrated for the three methods at baseline (90-92.2\% for T. trichiura, 41.1-52.8\% for hookworm, 32.9-37.2\% for A. lumbricoides); FLOTAC was the most sensitive method at baseline and follow-up. Albendazole showed high cure rate (CR) against A. lumbricoides (90-97\%), moderate CR against hookworm (63-72\%) and very low CR against T. trichiura (6-9\%), regardless of the technique used. Egg counts (eggs per gram) at baseline were similar for A. lumbricoides and for hookworm among the three methods, and higher using McMaster and Kato-Katz compared with FLOTAC for T. trichiura. All methods were similar for hookworm and A. lumbricoides egg reduction rate (ERR) estimation, but Kato-Katz indicated a significantly higher ERR than MCMaster and FLOTAC for T. trichiura. Preserved stool samples revealed consistently lower FECs at baseline and follow-up for all STHs.
\end{abstract}

Conclusion: Further development and validation of standard protocols for anthelminthic drug efficacy evaluation must be pursued.

Keywords: Soil-transmitted helminths, Diagnostic accuracy, Kato-Katz, McMaster, FLOTAC, Drug efficacy

\section{Introduction}

Preventive chemotherapy, using single oral doses of anthelminthic drugs (e.g. albendazole, mebendazole and praziquantel) is the current backbone of the global strategy to control soil-transmitted helminthiasis, schistosomiasis and other neglected tropical diseases. $^{1,2}$ The decision how, when and for how long to implement preventive chemotherapy is based on some kind of community diagnosis (e.g. questionnaire surveys for urogenital schistosomiasis, and qualitative or quantitative techniques for measuring excreted helminth eggs in faeces for intestinal schistosomiasis and soil-transmitted helminthiasis). ${ }^{3}$ It should be noted, however, that large-scale preventive chemotherapy in humans may trigger drug resistance, as has been observed in the veterinary field. ${ }^{4}$ Hence, rigorous drug efficacy monitoring with highly sensitive and quantitative diagnostic techniques is mandatory. ${ }^{5,6}$ Moreover, sensitive, inexpensive and field-applicable diagnostic tools are needed for monitoring progress of helminthiasis control programmes, especially with interventions going to scale and control programmes moving towards transmission control and local elimination..$^{-9}$

The most widely used diagnostic method for Schistosoma mansoni and soil-transmitted helminth (STH) infection is the 
Kato-Katz technique, ${ }^{10}$ but there are other copromicroscopic methods and their performance for assessing drug efficacy needs to be evaluated. The FLOTAC techniques are a series of copromicroscopic multivalent methods developed by veterinary parasitologists. ${ }^{11}$ An important feature of FLOTAC is that the diagnostic workup can be done with fixed faecal samples whereas more widely used copromicroscopic techniques, such as Kato-Katz and McMaster, are usually done with fresh stool. ${ }^{11,12}$ The use of fixed stool samples enables more flexible scheduling of daily laboratory activities and ensures the safety of the health staff who are exposed to health risks due to processing fresh faecal specimens. Several studies have shown that a single FLOTAC is more sensitive than multiple Kato-Katz thick smears for the diagnosis of the three common STHs, hookworm, Ascaris lumbricoides and Trichuris trichiura. ${ }^{12-15}$ Moreover, in terms of sensitivity, FLOTAC outperformed other techniques, such as the ether-concentration method on fixed stool ${ }^{12}$ and the McMaster technique using fresh stool. ${ }^{16}$

The McMaster egg counting technique is commonly used in veterinary parasitology and advocated by the World Association for the Advancement of Veterinary Parasitology in its guidelines for evaluating the efficacy of anthelminthic drugs in ruminants ${ }^{17}$ and for detection of anthelminthic drug resistance. ${ }^{18}$ The McMaster technique has been proposed as a valid alternative to the Kato-Katz for technique monitoring large-scale anthelminthic treatment programmes in humans. ${ }^{19,20}$

The purpose of this study was to determine the diagnostic accuracy of three copromicroscopic methods (Kato-Katz, FLOTAC and McMaster) for estimating drug efficacy against STH infections. The study was implemented on Pemba Island, where STHs are highly endemic and large-scale control efforts are underway. ${ }^{20,21}$ We included the McMaster technique since its diagnostic accuracy had not been investigated previously with formalin-fixed human faeces although it had been assessed for animal faeces. $^{22,23}$

\section{Materials and methods}

\section{Study design}

We designed a single-blinded intervention study to compare the diagnostic accuracy of the Kato-Katz, FLOTAC and McMaster techniques before and 21 days after administration of a single oral dose of albendazole (400 mg; GlaxoSmithKline Pharmaceuticals Ltd, Mumbai, India) against STH infections. The study was carried out between September and November 2009 on Pemba Island, one of two major islands of the Zanzibar archipelago, United Republic of Tanzania. STH infections are highly endemic in Zanzibar despite control efforts that have been in place since the mid-1990s emphasizing preventive chemotherapy with albendazole and mebendazole. ${ }^{24}$ Prevalence and intensity of STH infections, the primary outcome measures, were assessed before and 21 days post-treatment.

A total of 304 children from three primary schools (Kwale, Al Sadik and Ng'ombeni) were screened for the presence of STH infection during a cross-sectional baseline survey. Single stool samples were obtained, processed and examined using standard protocols for the Kato-Katz, McMaster and FLOTAC techniques. Children found positive for any STH species were given albendazole under direct medical supervision.
Twenty-one days after drug administration, children were again invited to provide a single stool sample. Faecal samples were quantitatively examined for STHs, using the same methods as during the baseline cross-sectional survey.

\section{Parasitological techniques}

Stool samples collected at baseline and at 21-day post-treatment follow-up were transferred to the Public Health Laboratory-Ivo de Carneri in Pemba. All samples were processed within a few hours. First, they were homogenized with a wooden spatula and divided into two aliquots, the first examined fresh and the second preserved in formalin 5\% (approximately $5 \mathrm{~g}$ of faeces in $15 \mathrm{ml}$ of formalin 5\%; dilution ratio 1:4).

Fresh stool samples at baseline and follow-up were examined using the Kato-Katz, McMaster and FLOTAC techniques, adhering to standard protocols. ${ }^{11,25,26}$ In brief, triplicate Kato-Katz thick smears using standard $41.7 \mathrm{mg}$ templates were prepared from each stool sample. Faecal egg counts (FEC) were estimated, counting the number of eggs of each STH per slide, and then calculating the arithmetic mean of the egg count from the three slides. FECs were then transformed into eggs per gram of stool (epg), using a multiplication factor of $24 .^{25}$ The term egg-positive refers to the presence of at least one helminth egg in any one of the three slides.

Stool samples were also examined in triplicate using the protocol of the modified McMaster method. ${ }^{26}$ In brief, approximately $2 \mathrm{~g}$ of faeces were placed in a plastic container and $30 \mathrm{ml}$ (dilution $1: 15$ ) of a saturated salt ( $\mathrm{NaCl}$; designated flotation solution no. 2 [FS2] with a specific gravity [s.g.] of 1.20) were added. ${ }^{11}$ Faecal samples were homogenized and poured three times through a tea strainer to remove the large debris. The filtrate was homogenized and McMaster counting chambers were filled using a Pasteur pipette. The slides were allowed to stand for $2 \mathrm{~min}$ before examination under a microscope at $100 \times$ magnification with all eggs in the two grids counted (volume $=2 \times 0.15 \mathrm{ml}=0.3 \mathrm{ml}$ ). For each STH species, we obtained FECs by multiplying the total number of eggs under the two grids by a factor of 50 (dilution/ volume $=15 / 0.3$ ).

Each stool sample was also examined with the FLOTAC basic technique which used FS2 as for the McMaster technique. The choice of the FS and dilution was preceded by a precalibration study using six different FSs: Sheather's sugar solution (FS1; s.g., 1.20); FS2 (see above); zinc sulfate (FS3; s.g., 1.20); sodium nitrate (FS4; s.g., 1.20); magnesium sulfate (FS6; s.g., 1.28); and zinc sulfate (FS7; s.g., 1.35) ${ }^{15}$ and four dilutions (1:10, 1:20, 1:25 and 1:30). The results of the precalibration study showed that, based on the clarity of reading and efficiency of bringing STH eggs into flotation, the most accurate diagnosis of STH infection was obtained using FS2 at a 1:25 dilution. Thus, for all the samples, FLOTAC was performed as follows. Five grams of faeces were weighed into a beaker and $120 \mathrm{ml}$ of tap water added, in order to obtain a 1:25 dilution. The samples were homogenized with a wooden spatula and filtered through a tea strainer and $11 \mathrm{ml}$ of the homogenized suspension were transferred into a test tube and centrifuged for 3 min at $170 \mathrm{~g}$ in a Hettich EBA Centrifuge (Tuttlingen, Germany). After centrifugation the supernatant was discarded and $11 \mathrm{ml}$ of FS2 were added, the sample was homogenized, and $5 \mathrm{ml}$ of the suspension were transferred into each of the two chambers of the FLOTAC apparatus. The FLOTAC apparatus was then centrifuged for $5 \mathrm{~min}$ at $120 \mathrm{~g}$ in a Hettich 
Universal 320 Centrifuge (Tuttlingen, Germany) and the apical portion of the floating suspension translated and examined under a microscope at 100x magnification. Helminth eggs were enumerated under the observation grids of both chambers and multiplied by a factor of 2.5 (dilution/volume $=25 / 10$ ) to obtain the epg for each STH species.

For quality control, a random sample of $10 \%$ of the Kato-Katz, MCMaster and FLOTAC slides were re-examined by a senior technician. This allowed rigorous evaluation of the accuracy of the diagnosis of STHs and the precision of the egg counts.

The stool samples preserved in formalin 5\% were shipped to Naples, Italy. The samples were stored for about 6 months and examined using the McMaster and FLOTAC protocols detailed above. Experienced laboratory technicians at the University of Naples Federico II carried out the microscopic examination. The technicians were blinded for the prior results obtained on the fresh stool samples in Pemba.

\section{Statistical analysis}

Data were entered into a Microsoft Excel 10.0 spreadsheet (Microsoft Corp., Redmond, WA, USA) and validated using EpiInfo software 3.3.2 (CDC, Atlanta, GA, USA). Statistical analyses were performed using STATA 10.0 (Stata Corp., College Station, TX, USA). Analyses were restricted to children who were diagnosed with a STH infection at baseline who were given albendazole and followed-up at 21 days post-treatment. The efficacy of the treatment was measured by a reduction in prevalence and intensity for each of the three helminth species separately. Infection intensity was indirectly measured by the mean FEC expressed as epg. The epgs calculated before and after treatment were used to provide an estimate of the egg reduction rate (ERR). Cure rate (CR) was calculated for each diagnostic method, based on the proportion of children who were found positive for STHs at the baseline cross-sectional survey who became eggnegative 21 days post-treatment. CRs as obtained by the different diagnostic methods used were compared by hypothesis testing and calculating $95 \%$ CIs.

The mean change in FECs before and after treatment was compared using a two-sample t-test. Although the change in FECs is not normally distributed, there are biological justifications for using the arithmetic mean rather than the median or a transformation of the data and corresponding formal analysis such as the Wilcoxon rank-sum test. ${ }^{27,28}$ Sensitivity and negative predictive value (NPV) were calculated for each of the three copromicroscopic techniques, considering the combined results of the three techniques as the diagnostic 'gold' standard. The statistical difference of the mean FEC was calculated with ANOVA, in conjunction with the Bonferroni test. Agreement between the three methods (i.e. Kato-Katz vs FLOTAC; Kato-Katz vs McMaster; and FLOTAC vs McMaster) was assessed using the $\mathrm{k}$ statistic. ${ }^{29}$ The $\mathrm{k}$ measures were interpreted as follows: 0, no agreement; 0.01-0.20, poor agreement; $0.21-$ 0.40, fair agreement; 0.41-0.60, moderate agreement; 0.61-0.80, substantial agreement; and $0.81-1.0$, nearly perfect agreement. ${ }^{30}$

\section{Ethical considerations}

The study is registered with Current Controlled Trials [identifier: ISRCTN90088840].

On the day prior to the scheduled treatment date, children eligible to participate in the study were given an informed consent letter in the local language (Kiswahili) to take home and to be signed by their parent or guardian. All children who were found with STHs in their stool at the end of the study received a second dose of albendazole (400 $\mathrm{mg}$ ) as per national guidelines.

\section{Results}

\section{Population characteristics}

Of 304 children screened at the baseline cross-sectional survey, 160 were girls (52.6\%). The mean age of participating children was 10 years (range $6-14$ ). At pretreatment screening, 278/304 (91.4\%) children were found positive for any STH infection. Among them, 238/278 (85.6\%) gave a second stool sample 21 days after drug administration. Seven samples were of insufficient quantity for being preserved for follow-up McMaster and FLOTAC examination. Analysis was performed on the remaining 231 children with a STH infection at baseline, who were treated with albendazole and who provided a sufficiently large stool sample that allowed re-examination by different methods, including formalin preservation for subsequent laboratory workup.

Results of quality control on random samples of $10 \%$ of KatoKatz, McMaster and FLOTAC slides confirmed all original counts.

\section{Diagnostic accuracy in estimating drug efficacy}

Table 1 summarizes the observed CRs for the three STH infections, stratified by the diagnostic technique used. Regardless of the diagnostic method used and whether stool samples were examined fresh or fixed in formalin, a single dose of albendazole $(400 \mathrm{mg})$ resulted in high CR against $A$. lumbricoides (Kato-Katz 97.4\% using fresh samples; FLOTAC $97.7 \%$ and $89.0 \%$, McMaster $97.4 \%$ and $96.1 \%$ on fresh and preserved samples, respectively), moderate CR against hookworm (Kato-Katz 79.0\%; FLOTAC $72.1 \%$ and $63.2 \%$, McMaster $78.4 \%$ and $73.1 \%$ on fresh and preserved samples, respectively), but only very low CR against $T$. trichiura (Kato-Katz 7.7\%; FLOTAC 6.1\% and 5.7\%, McMaster $6.7 \%$ and $8.8 \%$ on fresh and preserved samples, respectively).

Table 2 shows FECs before and after albendazole treatment and the observed ERRs. While consistently very high ERRs were observed against A. lumbricoides (Kato-Katz 99.3\%; FLOTAC and McMaster $99.9 \%$ on both fresh and preserved samples), considerably lower and more heterogeneous ERRs were observed against hookworm (Kato-Katz 90.7\%; FLOTAC 83.7\% and 57.1\%, McMaster $92.2 \%$ and $68.8 \%$ on fresh and preserved samples, respectively), and particularly against T. trichiura (Kato-Katz 50.3\%; FLOTAC 0 and $14.7 \%$, McMaster $20.9 \%$ and $19.7 \%$ on fresh and preserved samples, respectively). Preserving stool samples in formalin resulted in considerably lower FECs, with the most dramatic effect observed on quantification of hookworm eggs.

\section{Diagnostic sensitivity}

As summarized in Table 3, the prevalence of T. trichiura, hookworm and $A$. lumbricoides, according to our diagnostic 'gold' standard (i.e. positive result in at least one of the three methods used) was $95.2 \%$ (220/231), 65.4\% (151/231) and 45.5\% (105/231), respectively.

We found consistently high sensitivity for the diagnosis of T. trichiura regardless of the method used (ranging from $92.7 \%$ with McMaster on preserved samples to $96.8 \%$ with FLOTAC on 
Table 1. Number of children infected with soil-transmitted helminths at baseline and at 21-day post-treatment follow-up surveys, and observed cure rates, among 231 school-aged children on Pemba Island, diagnosed by three copromicroscopic methods

\begin{tabular}{|c|c|c|c|c|c|c|c|c|c|}
\hline \multirow[t]{2}{*}{$\begin{array}{l}\text { Diagnostic } \\
\text { method }^{a}\end{array}$} & \multicolumn{3}{|c|}{ No. of positive children at baseline } & \multicolumn{3}{|c|}{$\begin{array}{l}\text { No. of positive children at post-treatment } \\
\text { follow-up }\end{array}$} & \multicolumn{3}{|c|}{ Cure rate $(95 \% \mathrm{CI})$} \\
\hline & $\begin{array}{l}\text { Trichuris } \\
\text { trichiura }\end{array}$ & Hookworm & $\begin{array}{l}\text { Ascaris } \\
\text { lumbricoides }\end{array}$ & $\begin{array}{l}\text { Trichuris } \\
\text { trichiura }\end{array}$ & Hookworm & $\begin{array}{l}\text { Ascaris } \\
\text { lumbricoides }\end{array}$ & $\begin{array}{l}\text { Trichuris } \\
\text { trichiura }\end{array}$ & Hookworm & $\begin{array}{l}\text { Ascaris } \\
\text { lumbricoides }\end{array}$ \\
\hline Kato-Katz & 209 & 95 & 76 & 193 & 20 & 2 & $7.7(4.6-12.4)$ & $79.0(69.1-86.4)$ & $97.4(90.0-99.5)$ \\
\hline McMaster (fresh) & 208 & 102 & 78 & 194 & 22 & 2 & $6.7(3.9-11.3)$ & $78.4(69.0-85.7)$ & $97.4(90.0-99.5)$ \\
\hline $\begin{array}{l}\text { McMaster } \\
\quad \text { (preserved) }\end{array}$ & 204 & 78 & 76 & 186 & 21 & 3 & $8.8(5.5-13.8)$ & $73.1(61.6-82.2)$ & $96.1(88.1-99.0)$ \\
\hline FLOTAC (fresh) & 213 & 122 & 86 & 200 & 34 & 2 & $6.1(3.4-10.5)$ & $72.1(63.2-79.7)$ & $97.7(91.1-99.6)$ \\
\hline $\begin{array}{l}\text { FLOTAC } \\
\text { (preserved) }\end{array}$ & 211 & 106 & 82 & 199 & 39 & 9 & $5.7(3.1-10.0)$ & $63.2(53.2-72.2)$ & $89.0(79.7-94.6)$ \\
\hline
\end{tabular}

${ }^{a}$ Stool samples collected at baseline and at follow-up were divided into two aliquots: one was examined as a fresh sample and the other preserved in formalin $5 \%$ and examined approximately 6 months later.

Table 2. Faecal egg counts at baseline and at 21-day post-treatment follow-up surveys, and observed egg reduction rates, for soil-transmitted helminths among 231 school-aged children on Pemba Island, diagnosed by three copromicroscopic methods

\begin{tabular}{|c|c|c|c|c|c|c|c|c|c|}
\hline \multirow{2}{*}{$\begin{array}{l}\text { Diagnostic } \\
\text { method }^{a}\end{array}$} & \multicolumn{3}{|c|}{ epg at baseline } & \multicolumn{3}{|c|}{ epg at post-treatment follow-up } & \multicolumn{3}{|c|}{ Egg reduction rate (95\% CI) } \\
\hline & $\begin{array}{l}\text { Trichuris } \\
\text { trichiura }\end{array}$ & Hookworm & $\begin{array}{l}\text { Ascaris } \\
\text { lumbricoides }\end{array}$ & $\begin{array}{l}\text { Trichuris } \\
\text { trichiura }\end{array}$ & Hookworm & $\begin{array}{l}\text { Ascaris } \\
\text { lumbricoides }\end{array}$ & $\begin{array}{l}\text { Trichuris } \\
\text { trichiura }\end{array}$ & Hookworm & $\begin{array}{l}\text { Ascaris } \\
\text { lumbricoides }\end{array}$ \\
\hline Kato-Katz & $798^{*}$ & $193^{*}$ & $3697^{*}$ & $397^{\dagger}$ & $18^{*}$ & $25^{*}$ & $50.3(46.7-53.8)$ & $90.7(85.4-94.2)$ & $99.3(99.0-99.6)$ \\
\hline McMaster (fresh) & $954^{*}$ & $242^{*}$ & $2689^{*}$ & $755^{*}$ & $19^{*}$ & $2^{*}$ & $20.9(18.3-23.6)$ & $92.2(87.8-95.1)$ & $99.9(99.7-100)$ \\
\hline $\begin{array}{l}\text { McMaster } \\
\quad \text { (preserved) }\end{array}$ & $351^{\dagger}$ & $32^{\dagger}$ & $1491^{\dagger}$ & $282^{\dagger}$ & $10^{*}$ & $2^{*}$ & $19.7(15.7-24.3)$ & $68.8(49.9-83.3)$ & $99.9(99.7-100)$ \\
\hline FLOTAC (fresh) & $540^{\dagger}$ & $129 *$ & $2275^{*}$ & $559 *$ & $21^{*}$ & $2^{*}$ & 0 & $83.7(76.0-89.4)$ & $99.9(99.7-100)$ \\
\hline $\begin{array}{l}\text { FLOTAC } \\
\text { (preserved) }\end{array}$ & $464^{\dagger}$ & $35^{\dagger}$ & $1841^{*}$ & $396^{+}$ & $15^{*}$ & $2^{*}$ & $14.7(11.6-18.3)$ & $57.1(39.5-73.2)$ & $99.9(99.7-100)$ \\
\hline
\end{tabular}

epg: eggs per gram given as arithmetic mean; for each column epg values containing the same symbol are not significantly different, epg values containing different symbols are significantly different $(p<0.05)$.

${ }^{a}$ Stool samples collected at baseline and at follow-up were divided into two aliquots: one was examined as a fresh sample and the other preserved in formalin $5 \%$ and examined approximately 6 months later. 
Table 3. Prevalence, sensitivity and negative predictive value (NPV) at baseline and at 21-day post-treatment follow-up surveys among 231 school-aged children on Pemba Island, using three copromicroscopic methods for the diagnosis of soil-transmitted helminths

\begin{tabular}{|c|c|c|c|c|c|c|}
\hline \multirow[t]{2}{*}{ Diagnostic method ${ }^{a}$} & \multicolumn{3}{|l|}{ Baseline } & \multicolumn{3}{|c|}{ Post-treatment follow-up } \\
\hline & Prevalence $^{\mathrm{b}}(95 \% \mathrm{CI})$ & Sensitivity (95\% CI) & NPV $(95 \%$ CI) & Prevalence $^{\mathrm{b}}(95 \% \mathrm{CI})$ & Sensitivity (95\% CI) & NPV $(95 \%$ CI $)$ \\
\hline \multicolumn{7}{|l|}{ Trichuris trichiura } \\
\hline 'Gold' standard' & $95.2(91.4-97.5)$ & & & $91.8(87.3-94.8)$ & & \\
\hline Kato-Katz & $90.5^{*}(85.8-93.8)$ & $95.0(91.0-97.4)$ & $50.0(28.8-71.2)$ & $85.3^{*}(79.9-89.5)$ & 92.9 (88.4-95.9) & $55.9(38.1-72.4)$ \\
\hline McMaster (fresh) & $90.0^{*}(85.3-93.5)$ & $94.6(90.4-97.0)$ & $47.8(27.4-68.9)$ & $86.2^{*}(80.9-90.2)$ & $93.9(89.5-96.6)$ & $59.4(40.8-75.8)$ \\
\hline McMaster (preserved) & $88.3^{\dagger}(83.3-92.0)$ & $92.7(88.2-95.7)$ & $40.7(23.0-61.0)$ & $83.6^{\dagger}(78.0-88.0)$ & $91.0(86.2-94.4)$ & $50.0(33.7-66.3)$ \\
\hline FLOTAC (fresh) & $92.2^{*}(87.8-95.2)$ & $96.8(93.3-98.6)$ & $61.1(36.1-81.7)$ & $88.3^{*}(83.3-92.0)$ & $96.2(92.4-98.2)$ & $70.4(49.7-85.5)$ \\
\hline FLOTAC (preserved) & $91.3^{*}(86.8-94.5)$ & $95.9(92.1-98.0)$ & $55.0(32.1-76.2)$ & $87.4^{*}(82.3-91.3)$ & $95.3(91.2-97.6)$ & $65.5(45.7-81.4)$ \\
\hline \multicolumn{7}{|l|}{ Hookworm } \\
\hline 'Gold' standard' & $65.4(58.8-71.4)$ & & & $25.5(20.1-31.7)$ & & \\
\hline Kato-Katz & $41.1^{\dagger}(34.8-47.8)$ & $62.9(54.6-70.5)$ & $58.8(50.0-67.1)$ & $9.5^{\ddagger}(6.2-14.3)$ & $37.3(25.3-50.9)$ & $82.3(76.3-87.1)$ \\
\hline McMaster (fresh) & $44.2^{\dagger}(37.7-50.8)$ & $67.6(59.4-74.8)$ & $62.0(53.0-70.3)$ & $11.7^{\ddagger}(8.0-16.7)$ & $45.8(32.9-59.2)$ & $84.3(78.4-88.9)$ \\
\hline McMaster (preserved) & $33.8^{\dagger}(27.8-40.3)$ & $51.7(43.4-59.8)$ & $52.3(44.1-60.4)$ & $11.7^{\ddagger}(8.0-16.7)$ & $45.8(32.9-59.2)$ & $84.3(78.4-88.9)$ \\
\hline FLOTAC (fresh) & $52.8^{*}(46.2-59.4)$ & $80.8(73.4-86.6)$ & $73.4(63.9-81.2)$ & $16.0^{\dagger}(11.7-21.5)$ & $62.7(49.1-74.7)$ & $88.7(83.1-92.6)$ \\
\hline FLOTAC (preserved) & $45.9^{\dagger}(39.4-52.5)$ & $70.2(62.1-77.2)$ & $64.0(54.9-72.3)$ & $21.2^{*}(16.2-27.2)$ & $83.1(70.6-91.2)$ & $94.5(89.8-97.2)$ \\
\hline \multicolumn{7}{|l|}{ Ascaris lumbricoides } \\
\hline 'Gold' standard' & $45.5(38.9-52.1)$ & & & $7.4(4.5-11.7)$ & & \\
\hline Kato-Katz & $32.9^{\dagger}(27.0-39.4)$ & $72.4(67.2-80.4)$ & $81.3(74.1-86.9)$ & $0.9^{\dagger}(0.2-3.4)$ & $11.8(2.1-37.8)$ & $93.4(89.2-96.2)$ \\
\hline McMaster (fresh) & $33.8 *(27.8-40.3)$ & $74.3(64.7-82.1)$ & $82.4(75.2-87.9)$ & $0.9^{+}(0.2-3.4)$ & $11.8(2.1-37.8)$ & $93.4(89.2-96.2)$ \\
\hline McMaster (preserved) & $32.9^{\dagger}(27.0-39.4)$ & $72.4(67.2-80.4)$ & $81.3(74.1-86.9)$ & $1.7^{\dagger}(0.6-4.7)$ & $23.5(7.8-50.2)$ & $94.3(90.2-96.8)$ \\
\hline FLOTAC (fresh) & $37.2^{*}(31.1-43.8)$ & $81.9(72.9-88.5)$ & 86.9 (80.1-91.7) & $1.3^{\dagger}(0.3-4.1)$ & $17.6(4.7-44.2)$ & $93.9(89.7-96.5)$ \\
\hline FLOTAC (preserved) & $35.5^{*}(29.4-42.1)$ & $78.1(68.8-85.3)$ & $84.6(77.5-89.8)$ & $6.1^{*}(3.5-10.2)$ & $82.4(55.8-95.3)$ & $98.6(95.7-99.6)$ \\
\hline
\end{tabular}


Table 4. k agreement between three copromicroscopic methods for the diagnosis of soil-transmitted helminths at baseline and at 21-day posttreatment follow-up surveys among 231 school-aged children on Pemba Island

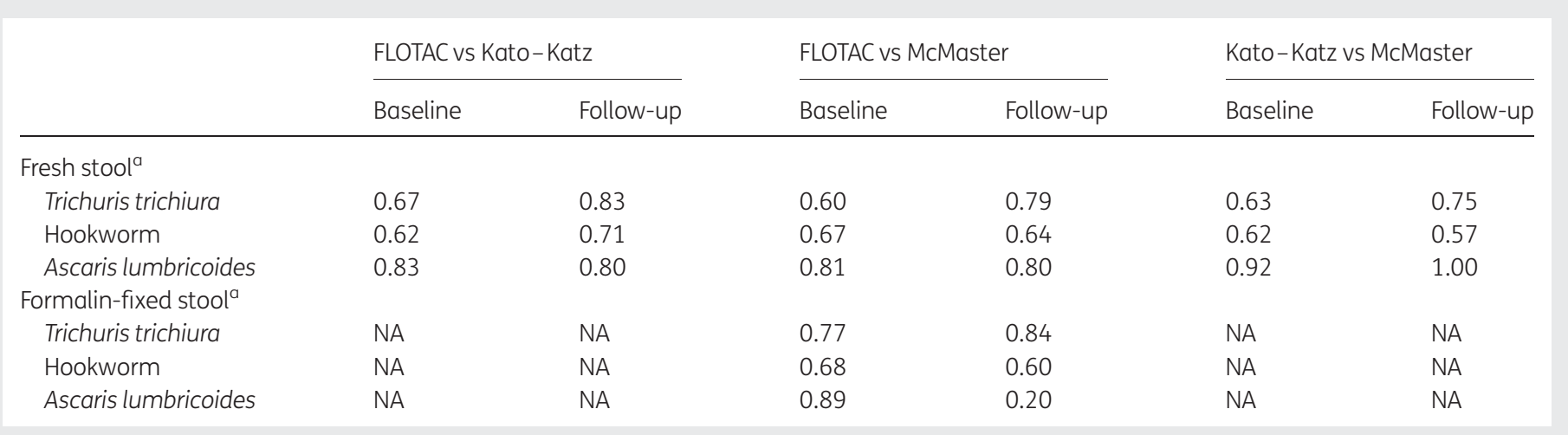

NA: not applicable (preserved samples were examined using the FLOTAC and McMaster techniques only).

${ }^{a}$ Stool samples collected at baseline and at follow-up were divided into two aliquots: one was examined as a fresh sample and the other preserved in formalin $5 \%$ and examined approximately 6 months later.

fresh samples). Table 4 shows the k agreement between the three methods. For the diagnosis of $T$. trichiura using fresh stool at the baseline cross-sectional survey, agreement was moderate $(k=$ 0.60-0.67), while it was substantial at the 21-day treatment follow-up $(k=0.75-0.83)$. The agreement between the FLOTAC and McMaster methods for the diagnosis of $T$. trichiura using formalin-fixed faeces at baseline and at 21-day follow-up was substantial $(k=0.77-0.84)$.

FLOTAC showed a significantly higher sensitivity for hookworm diagnosis $(80.8 \%$ on fresh samples, $70.2 \%$ on preserved samples) compared with the other two methods (Kato-Katz, $62.9 \%$; McMaster $67.6 \%$ on fresh samples and $51.7 \%$ on preserved samples). The $\mathrm{k}$ agreement between the three methods for the diagnosis of hookworm at baseline was substantial $(k=0.62-$ 0.67). Similar agreements were found at treatment follow-up $(k=0.57-0.71)$. Similarly, the $k$ agreement between FLOTAC and McMaster for the diagnosis of hookworm using preserved samples at baseline was substantial $(k=0.68)$, while it was only moderate at the post-treatment follow-up survey $(k=0.60)$.

For A. lumbricoides diagnosis, FLOTAC showed a somewhat higher sensitivity $(81.9 \%$ on fresh samples, $78.1 \%$ on preserved samples) compared with Kato-Katz (72.4\%) and McMaster (74.3\% on fresh samples, $72.4 \%$ on preserved samples). The $\mathrm{k}$ values between the three methods for the diagnosis of A. lumbricoides at baseline showed nearly perfect agreement $(k=0.81-0.92)$. Similar results were obtained at the treatment follow-up survey $(k=0.80-1.00)$. The $k$ agreement between FLOTAC and MCMaster for the diagnosis of $A$. lumbricoides using fixed faeces at baseline was nearly perfect ( $k=0.89$ ), while only poor agreement was found at treatment follow-up $(k=0.20)$.

At the 21-day treatment follow-up, FLOTAC consistently showed the highest sensitivity for diagnosis of all three STHs.

\section{Discussion}

To our knowledge, this is the first comparison of three copromicroscopic methods for assessing anthelminthic drug efficacy against the three common species of STH infections in an area highly endemic for these intestinal nematodes. ${ }^{20,21}$ Moreover, our study was the first attempt to determine the effect of preserving stool samples in formalin prior to laboratory workup using the McMaster and FLOTAC techniques.

Our observed CRs confirm that a single oral dose of albendazole (400 mg) shows high, moderate and poor efficacy against A. lumbricoides, hookworm and T. trichiura, respectively. ${ }^{31-34} \mathrm{Im}-$ portantly though, CRs calculated by the three methods were not significantly different. Data on CR from formalin-fixed samples for the MCMaster and FLOTAC methods indicate a similar performance to the ones with fresh samples.

Results from the ERRs on fresh samples were similarly high for A. lumbricoides regardless of the diagnostic method used, however, the ERRs were lower for hookworm when assessed by FLOTAC compared with the other two methods ( $84 \%$ vs $>90 \%$ ), and significantly different for $T$. trichiura for all methods. For T. trichiura, the ERR for Kato-Katz ( 50\%) confirms previous data, ${ }^{5,20}$ while the ERR for McMaster ( 20\%) shows lower efficacy compared with other drug intervention studies using the same method. ${ }^{20,28}$ With regard to $A$. lumbricoides and hookworm, FECs at the 21-day treatment follow-up were significantly $(p<0.05)$ lower than those at baseline with any of the copromicroscopic techniques used and considering both fresh and fixed stool samples. For T. trichiura, FECs at follow-up were significantly ( $p<$ 0.05) lower than those at baseline only when using Kato-Katz, whereas no significant difference was found in FECs at baseline and follow-up using McMaster and FLOTAC (both fresh and preserved samples). The finding of no changes in ERR for T. trichiura infection with FLOTAC and MCMaster using fresh faecal samples is surprising and of considerable concern. The following points are offered for consideration. First, the differences in observed ERRs may be explained by Kato-Katz thick smear examinations overestimating $\mathrm{FECs}^{14}$ or flotation methods (McMaster and FLOTAC) underestimating FECs. Second, it is conceivable that bringing all T. trichiura eggs into flotation is much harder at high infection intensities, while all or most of the eggs would float in light-intensity 
infections. Third, in the Kato-Katz method, the faeces are weighed (by volume) after sieving the sample through a mesh thereby eliminating fibres and other debris, and therefore concentrating the eggs in the faecal material. Fourth, by weighing with electronic scale, the stool volume measured by the standard template of $41.7 \mathrm{mg}$ revealed considerable variation (e.g. 95\% CI 14.3$66.7 \mathrm{mg}){ }^{19}$ These points corroborate with the observation that for all three species of STHs, the FECs obtained by Kato-Katz and McMaster are considerably higher when compared with FLOTAC. ${ }^{14,35}$

The differences observed in the ERRs of T. trichiura by the three methods have important implications for assessing anthelminthic drug efficacy. Despite very low CR, a variation between diagnostic methods of reduction in FECs from nil by FLOTAC to $50 \%$ by Kato-Katz, suggests that further development and rigorous validation of standardized procedures for diagnostic methods for this parasite is a pressing need. In addition, the preserved stool samples demonstrate consistently lower FEC both at baseline and at treatment follow-up for all three STHs investigated. Ascaris lumbricoides FECs in preserved samples were $50 \%$ less than the fresh samples for McMaster and 35\% less for FLOTAC; T. trichiura FECs in preserved samples were $32 \%$ less than the fresh samples for McMaster, and 22\% less for FLOTAC; hookworm FECs in preserved samples were $21 \%$ less than the fresh samples for McMaster and $12 \%$ less for FLOTAC. These data confirm the finding that with any preservatives, mean helminth egg recovery decreases over time. ${ }^{14,20,36}$ In this study, samples fixed in 5\% formalin were read after approximately 6 months of preservation, and while sensitivity and CR did not differ from those calculated on fresh faecal samples, this seemed to be too long a time interval for a reliable mean egg recovery compared with fresh samples.

We found that the sensitivity of Kato-Katz and McMaster in light-intensity and moderate-intensity infections at the baseline cross-sectional survey were similar ( $95 \%$ for $T$. trichiura, $\sim 75 \%$ for A. lumbricoides and $\sim 70 \%$ for hookworm). The sensitivity of the FLOTAC was consistently higher, particularly for hookworm, where the difference showed statistical significance. This result is in agreement with previous studies that suggest that the sensitivity of the Kato-Katz technique in determining STH infection is low, specially for hookworm detection. ${ }^{37}$

After treatment, when most infections had come down to low levels, the sensitivity of Kato-Katz and McMaster were considerably lower than before treatment. In contrast, the sensitivity of FLOTAC remained high (88-98\%). For the diagnosis of hookworm, FLOTAC picked up significantly more infections than the Kato-Katz technique. Our findings therefore confirm that a single FLOTAC is more sensitive than triplicate Kato-Katz thick smears and triplicate McMaster tests, particularly for hookworm infection. ${ }^{12,13,31}$ Although FLOTAC is a more complex and time-consuming method, it should be taken into consideration that timing and feasibility of performing multiple Kato-Katz and multiple McMaster vs a single FLOTAC might tip the balance towards FLOTAC. ${ }^{16,38}$ Assessment by the $\mathrm{k}$ statistic confirmed the moderate-to-substantial agreement among the three copromicroscopic methods at baseline and at follow-up surveys with fresh samples, but are not consistent with fixed samples probably being influenced by the reduction in FECs in preserved samples. Data on sensitivity and NPV showed no significant difference for both FLOTAC and McMaster between fresh and formalin-fixed stool samples. The advantages of working with fixed faecal material are ensuring safety of the diagnostic method by protecting the health staff, and allowing a more flexible planning of the workload in the laboratory. ${ }^{11,14}$ On the other hand, the somewhat reduced sensitivity, and particularly the considerably lower FECs are important shortcomings of preservation of stool samples, especially when samples are stored for several months.

When dealing with drug efficacy, treatment failures or reduced efficacy must be differentiated from reinfection. Drug efficacy in this study was assessed 3 weeks from treatment and this interval would exclude reinfection of any STH species. On the other hand, parasitological indicators that assess effectiveness or impact of control intervention are assessed just before the next round of preventive chemotherapy (6 or 12 months after previous treatment), as shown in studies that assess the impact of control intervention, and must take reinfection into account. ${ }^{37,39,40}$ Reinfection after treatment is the rule in helminth control based on preventive chemotherapy, depending on the intensity of transmission. ${ }^{41} \mathrm{Re}-$ cently a model has been proposed to assess decreasing trend in prevalence of STH infections, depending on pretreatment infection level and taking into account the frequency of reinfection. ${ }^{42}$ The diagnostic methods evaluated in this study could be used for both evaluating drug efficacy as well as for monitoring impact of control programmes.

\section{Limitations}

Our study has some limitations and drawbacks. Comparisons between baseline and post-treatment and between fresh and fixed samples were possible only on 231 samples of 304 samples collected at the baseline survey, due to the dropout rate at followup and insufficient amount of stool in a few samples. Moreover, samples fixed with formalin $5 \%$ had been analysed after approximately 6 months of preservation. Different studies have demonstrated that the prolonged preservation of stool in fixatives might destroy the eggs, especially fragile hookworm eggs. ${ }^{13,14}$

\section{Conclusion}

The FLOTAC basic technique showed higher sensitivity for all STH species considered, especially so for low-intensity infections. Hence, FLOTAC may become the method of choice in areas where STH prevalence and intensities are low as in the elimination phase of control programmes. When assessing drug efficacy, CR is not affected by the diagnostic method used, while ERR showed important variations that warrant further scientific inquiry.

Authors' contributions: MA, LR, JU and GC conceived and designed the study; SS, MEM, MP, MPM and VM analysed the data; MA, LR, SMAl and SMAm interpreted the data; SS, MEM, MP, MPM, VM, SMAl and SMAm drafted the manuscript; MA, LR, JU and GC critically revised the manuscript for intellectual content. All authors read and approved the final version of the manuscript. MA, JU and GC are guarantors of the paper.

Acknowledgements: This study would not have been possible without the competence and dedication of the laboratory technicians from the Parasitology Team of the Public Health Laboratory-Ivo de Carneri, Pemba, Tanzania. Special thanks go to the teachers and children of Kwale, Al Sadik and Ng'ombeni primary schools on Pemba Island for their enthusiastic participation. 
Funding: This work was supported by the University of Naples Federico II (Italy). SS was partially funded by a scholarship from the University of Torino (Italy).

Competing interests: $\mathrm{GC}$ is the inventor and current patent holder of the FLOTAC apparatus. If the currently ongoing development and validation of the FLOTAC apparatus is successful, the method will be licensed free of charge to the $\mathrm{WHO}$ and interested public non-commercial research centres. All other authors have no competing interests.

Ethical approval: This study was approved by the ethics committees of Basel [EKBB; reference no. 278/09] and the Ministry of Health, Zanzibar [ZAMREC; reference no. 0001/09]. The study is registered with Current Controlled Trials [identifier: ISRCTN90088840].

\section{References}

1 WHO. Preventive chemotherapy in human helminthiasis. Coordinated use of anthelminthic drugs in control interventions: a manual for health professionals and programme managers. Geneva: World Health Organization; 2006.

2 Hotez PJ, Molyneux DH, Fenwick A et al. Control of neglected tropical diseases. New Engl J Med 2007;357:1018-27.

3 WHO. Prevention and control of schistosomiasis and soil-transmitted helminthiasis. Report of a WHO Expert Committee. Geneva: World Health Organization; 2002, Technical Report Series No. 912.

4 Geerts S, Gryseels B. Drug resistance in human helminths: current situation and lessons from livestock. Clin Microbiol Rev 2000;13:207-22.

5 Albonico M, Engels D, Savioli L. Monitoring drug efficacy and early detection of drug resistance in human soil-transmitted nematodes: a pressing public health agenda for helminth control. Int J Parasitol 2004;34:1205-10.

6 Knopp S, Speich B, Hattendorf J et al. Diagnostic accuracy of Kato-Katz and FLOTAC for assessing anthelmintic drug efficacy. PLoS Negl Trop Dis 2011;5:e1036.

7 Bergquist R, Johansen MV, Utzinger J. Diagnostic dilemmas in helminthology: what tools to use and when? Trends Parasitol 2009;25:151-6.

8 Knopp S, Stothard JR, Rollinson D et al. From morbidity control to transmission control: time to change tactics against helminths on Unguja Island, Zanzibar. Acta Trop 2011;DOI:10.1016/j.actatropica. 2011.04.010.

9 Rollinson D, Knopp S, Levitz $S$ et al. Time to set the agenda for schistosomiasis elimination. Acta Trop 2012;DOI:10.1016/j.actatropica. 2012.04.013.

10 Katz N, Chaves A, Pellegrino J. A simple device for quantitative stool thick-smear technique in schistosomiasis mansoni. Rev Inst Med Trop São Paulo 1972;14:397-400.

11 Cringoli G, Rinaldi L, Maurelli MP, Utzinger J. FLOTAC: new multivalent techniques for qualitative and quantitative copromicroscopic diagnosis of parasites in animals and humans. Nat Protoc 2010;5:503-15.

12 Utzinger J, Rinaldi L, Lohourignon LK et al. FLOTAC: a new sensitive technique for the diagnosis of hookworm infections in humans. Trans R Soc Trop Med Hyg 2008;102:84-90.

13 Knopp S, Rinaldi L, Khamis IS et al. A single FLOTAC is more sensitive than triplicate Kato-Katz for the diagnosis of low-intensity soil-transmitted helminth infections. Trans R Soc Trop Med Hyg 2009;103:347-54.

14 Glinz D, Silué KD, Knopp S et al. Comparing diagnostic accuracy of Kato-Katz, Koga agar plate, ether-concentration, and FLOTAC for Schistosoma mansoni and soil-transmitted helminths. PLoS Negl Trop Dis 2010;4:e754.
15 Habtamu K, Degarege A, Ye-Ebiyo Y, Erko B. Comparison of the Kato-Katz and FLOTAC techniques for the diagnosis of soil-transmitted helminth infections. Parasitol Int 2011;60:398-402.

16 Levecke B, De Wilde N, Vandenhoute E, Vercruysse J. Field validity and feasibility of four techniques for the detection of Trichuris in simians: a model for monitoring drug efficacy in public health? PLoS Negl Trop Dis 2009;3:366-74

17 Wood IB, Amaral NK, Bairden $\mathrm{K}$ et al. World Association for the Advancement of Veterinary Parasitology (W.A.A.V.P.) second edition of guidelines for evaluating the efficacy of anthelmintics in ruminants (bovine, ovine, caprine). Vet Parasitol 1995;58:181-213.

18 Coles GC, Jackson F, Pomroy WE et al. The detection of anthelmintic resistance in nematodes of veterinary importance. Vet Parasitol 2006;136:167-85.

19 Levecke B, Behnke JM, Ajjampur SS et al. A comparison of the sensitivity and fecal egg counts of the McMaster egg counting and Kato-Katz thick smear methods for soil-transmitted helminths. PLoS Negl Trop Dis 2011;5:e1201.

20 Albonico M, Ame SM, Vercruysse J, Levecke B. Comparison of Kato-Katz thick smear and McMaster egg counting method for monitoring drug efficacy against soil-transmitted helminths in school children of Pemba Island, Tanzania. Trans R Soc Trop Med Hyg 2012;106:199-201.

21 Speich B, Ame SM, Ali SM et al. Efficacy and safety of nitazoxanide, albendazole, and nitazoxanide-albendazole against Trichuris trichiura infection: a randomized controlled trial. PLoS Negl Trop Dis 2012;6:e1685.

22 Cringoli G, Rinaldi L, Maurelli MPet al. Ancylostoma caninum: calibration and comparison of diagnostic accuracy of flotation in tube, McMaster and FLOTAC in faecal samples of dogs. Exp Parasitol 2011;128:32 - 7.

23 Rinaldi L, Coles GC, Maurelli MPet al. Calibration and diagnostic accuracy of simple flotation, McMaster and FLOTAC for parasite egg counts in sheep. Vet Parasitol 2011;177:345-52.

24 Albonico M, Stoltzfus RJ, Savioli L et al. A controlled evaluation of two school-based antihelmintic chemotherapy regimens on intensity of helminth infections. Int J Epidemiol 1999;28:591-6.

25 WHO. Bench aids for the diagnosis of intestinal parasites. Geneva: World Health Organization; 1994.

26 MAFF. Manual of veterinary parasitological laboratory techniques. London: Ministry of Agriculture, Fisheries and Food; 1986, p. 12-3.

27 Montresor A. Arithmetic or geometric means of eggs per gram are not appropriate indicators to estimate the impact of control measures in helminth infections. Trans R Soc Trop Med Hyg 2007;101:773-6.

28 Vercruysse J, Behnke JM, Albonico $M$ et al. Assessment of the anthelmintic efficacy of albendazole in school children in seven countries where soil-transmitted helminths are endemic. PLoS Negl Trop Dis 2011;5:e948.

29 Thrusfield M. Veterinary epidemiology. London: Blackwell; 1995.

30 Landis JR, Koch GG. The measurement of observer agreement for categorical data. Biometrics 1977;33:159-74.

31 Knopp S, Glinz D, Rinaldi L et al. FLOTAC: a promising technique for detecting helminth eggs in human faeces. Trans R Soc Trop Med Hyg 2009;103,1190-4.

32 Keiser J, Utzinger J. Efficacy of current drugs against soil-transmitted helminth infections: systematic review and meta-analysis. JAMA 2008;299:1937-48.

33 Keiser J, Utzinger J. The drugs we have and the drugs we need against major helminth infections. Adv Parasitol 2010;73:197-230.

34 Steinmann P, Utzinger J, Du ZW et al. Efficacy of single-dose and triple-dose albendazole and mebendazole against soil-transmitted helminths and Taenia spp.: a randomized controlled trial. PLoS One 2011;6:e25003. 
35 Cringoli G, Rinaldi L, Veneziano Vet al. The influence of flotation solution, sample dilution and the choice of McMaster slide area (volume) on the reliability of the McMaster technique in estimating the faecal egg counts of gastrointestinal strongyles and Dicrocoelium dendriticum in sheep. Vet Parasitol 2004;123:121-31.

36 Foreyt WJ. Recovery of nematode eggs and larvae in deer: evaluation of fecal preservation methods. Am J Vet Med Assoc 1986;189: $1065-7$.

37 Sanza M, Totanes FI, Chua PL VY Jr. Helminthiasis (STH) control in the Western Visayas Region of the Philippines from 2007 through 2011. Acta Trop 2013;127:112 - 7.

38 Speich B, Knopp S, Mohammed KA. Comparative cost assessment of the Kato-Katz and FLOTAC techniques for soil-transmitted helminth diagnosis in epidemiological surveys. Parasit Vectors 2010;3:71.
39 Sinuon M, Tsuyuoka R, Socheat D et al. Control of Schistosoma mekongi in Cambodia: results of eight years of control activities in the two endemic provinces. Trans R Soc Trop Med Hyg. 2007;101:34-9.

40 Mihrshahi S, Casey GJ, Montresor A et al. The effectiveness of 4 monthly albendazole treatment in the reduction of soil-transmitted helminth infections in women of reproductive age in Viet Nam. Int J Parasitol 2009;39:1037-43.

41 Jia TW, MelvilleS, Utzinger J et al. Soil-transmitted helminth reinfection after drug treatment: a systematic review and meta-analysis. PLoS Negl Trop Dis 2013; 6:e1621.

42 Montresor A, Gabrielli AF, Yajima A et al. Markov model to forecast the change in prevalence of soil-transmitted helminths during a control programme: a case study in Vietnam. Trans R Soc Trop Med Hyg 2013;107:313-8. 